\title{
MONTE CARLO INITIALIZATION FOR MULTI-SENSOR BEARING ONLY TRACKING
}

\author{
Alon Shalev Housfater, Xiao-Ping Zhang
}

\author{
Department of Electrical \& Computer Engineering \\ 350 Victoria Street \\ Ryerson University, Toronto, Canada \\ E-mail: $\{$ ashalev,xzhang $\} @$ ee.ryerson.ca
}

\author{
Yifeng Zhou \\ Defence R\&D Canada - Ottawa \\ 3701 Carling Avenue \\ Ottawa, Ontario K1A $0 \mathrm{Z4}$ \\ E-mail: yifeng.zhou@drdc-rddc.gc.ca
}

\begin{abstract}
A new algorithm for particle filter initialization for multisensor bearing only tracking is developed to enhance tracker performance and stability. Multiple bearing observations are used by a least squares technique to form multiple initial position estimates; these estimates are in turn used to compute the statistics of the initial state distribution. Simulated data is used to demonstrate the performance and efficiency of the algorithm by comparing the new initialization technique to a filter initialized with the true initial state.
\end{abstract}

Index Terms - Adaptive filters, Monte Carlo methods, Electronic countermeasures, Tracking filters

\section{INTRODUCTION}

Bearing only tracking is a highly nonlinear problem that can be effectively tackled using Sequential Monte Carlo methods such as the particle filter [3]. The particle filter is a nonlinear estimation technique which obtains a discrete approximation of the optimal filtering density. However, a direct application of the particle filter to bearing only tracking is problematic since incorrect filter initialization can cause divergent behavior. The past work on this topic focuses on strategies for initializing the extended Kalman filter (EKF) in modified polar coordinates domain [1] or by using a bank of range parameterized extended Kalman filters (RPEKF) [2]. In this paper, a new method for particle filter initialization is presented for the case of multiple bearing only sensors.

The classical algorithms for bearing only tracking, the EKF and RPEKF, operate by linearizing the nonlinear state space and then applying the well known Kalman filter to this linearized state space. The RPEKF algorithm deals with the initialization issue directly by assuming that the initial range of the target is known to be between two limits [2]. Next, the algorithm uses a geometric sequence to pick ranges between the two limits. Each EKF in the filter bank is initialized with a different initial range from the sequence and the first available bearing measurement. The convergence of each EKF is then examined after a few time iterations and diverging filters are removed from the filter bank. This initialization strategy can be viewed in probabilistic terms by noting that the initial state distribution is assumed to be a uniform distribution between the two known limits of the initial range. This uniform distribution is sampled in a deterministic manner using a geometric sequence where each sample is used by a different EKF.

The idea used by the RPEKF initialization can be expanded and generalized to a particle filter initialization strategy. We construct a Gaussian initial state distribution that approximates the true initial state distribution. Next, the particle filter samples from this state distribution. This initial sampling can be connected to the notion of multiple filters each initialized with a different range by noting that each particle can be considered a simple filter that is initialized with a different range that is randomly picked. Moreover, the deterministic sampling of the RPEKF is expanded to a stochastic sampling of the initial state distribution by the particle filter. The initialization algorithm is tested using simulated tracks, it is compared against an EKF algorithm which is initialized with the ground truth data. By observing the initial performance of both the EKF and the particle filter, it is clear that the newly developed initialization technique method performs well. Moreover, simulations indicate that the initialization method, on average, performs equally well as initializing the filter with the true initial state which is the best possible initialization.

\section{PROBLEM FORMULATION}

Consider a target moving in two dimensional space with position coordinates $\left(x_{n}, y_{n}\right)$ and velocity components $\left(v_{n}^{x}, v_{n}^{y}\right)$, assume that the target does not maneuver and follows a near constant velocity model [4]. Thus, one has state dynamics equation

$$
\mathbf{X}_{n}=\mathbf{\Phi}_{n} \mathbf{X}_{n-1}+\mathbf{\Gamma}_{n} \mathbf{V}_{n}
$$

Let the time difference between two successive measurements indexed by $n$ and $n-1$ be denoted as $\Delta t_{n}$, then the state and 
noise transition matrices are written,

$$
\Phi_{n}=\left(\begin{array}{cccc}
1 & \Delta t_{n} & 0 & 0 \\
0 & 1 & 0 & 0 \\
0 & 0 & 1 & \Delta t_{n} \\
0 & 0 & 0 & 1
\end{array}\right),
$$

and,

$$
\boldsymbol{\Gamma}_{n}=\left(\begin{array}{cc}
\Delta t_{n}^{2} / 2 & 0 \\
\Delta t_{n} & 0 \\
0 & \Delta t_{n}^{2} / 2 \\
0 & \Delta t_{n}
\end{array}\right)
$$

The state vector is written $\mathbf{X}_{n}=\left(x_{n}, y_{n}, v_{n}^{x}, v_{n}^{y}\right)^{T}$ and $\mathbf{V}_{n} \sim$ $\mathcal{N}\left(0, \boldsymbol{\Sigma}_{v}\right)$ is the state transition noise. Assume there are $K$ sensors, each one taking a noisy bearing measurement of the target's position. These sensors are modeled using the standard bearing only measurement model

$$
\mathbf{Y}_{n}=\left(\begin{array}{c}
Y_{n}^{1} \\
Y_{n}^{2} \\
\vdots \\
Y_{n}^{K}
\end{array}\right)=\left(\begin{array}{c}
\arctan \left(\frac{y_{n}-y_{s, n}^{1}}{x_{n}-x_{s, n}^{1}}\right) \\
\arctan \left(\frac{y_{n}-y_{s, n}^{2}}{x_{n}-x_{s, n}^{2}}\right) \\
\vdots \\
\arctan \left(\frac{y_{n}-y_{s, n}^{K}}{x_{n}-x_{s, n}^{K}}\right)
\end{array}\right)+\mathbf{W}_{n}
$$

Let the pair $\left(x_{s, n}^{i}, y_{s, n}^{i}\right)$ denote the cartesian position of the $i$ th sensor at time $n$. The observation noise is a $K$-dimensional Gaussian noise distribution, $\mathbf{W}_{n} \sim \mathcal{N}\left(M_{w}, \boldsymbol{\Sigma}_{w}\right)$. Refer to Figure 1 for a typical geometry of this problem. The final objective of tracking algorithms is to obtain the posteriori probability density function of the state given all past and present observations. We write this density function as $p\left(\mathbf{X}_{n} \mid \mathbf{Y}_{0: n}\right)$ where $\mathbf{Y}_{0: n}$ denotes all observations from the initial time instance to time instance $n$. In order for a tracking algorithm to operate, it must be first initialized using an initial estimate of the target position.

\section{PARTICLE FILTERS AND INITIAL STATE DISTRIBUTION}

A well known tracking algorithm that has shown good performance in dealing with nonlinear systems is the particle filter [3]. This algorithm is a statistical recursive filter that obtains an estimate in a Bayesian sense by computing the a posteriori probability density $p\left(\mathbf{X}_{n} \mid \mathbf{Y}_{0: n}\right)$. It turns out that this density cannot be computed analytically except for special state space models (such as the Kalman filter for the Gaussianlinear model). Therefore, the particle filter resorts to a Monte Carlo sampling strategy that allows to obtain a discrete approximation of the filtering probability density. The particle filter uses a set of $N$ simulated states, or particles, $\mathbf{X}_{n}^{i}$ and their corresponding weights $w_{n}^{i}$ for each time instance $n$ where $i$ indexes the particles. These particles, $\left\{w_{n}^{i}, \mathbf{X}_{n}^{i}\right\}_{i=1}^{N}$,

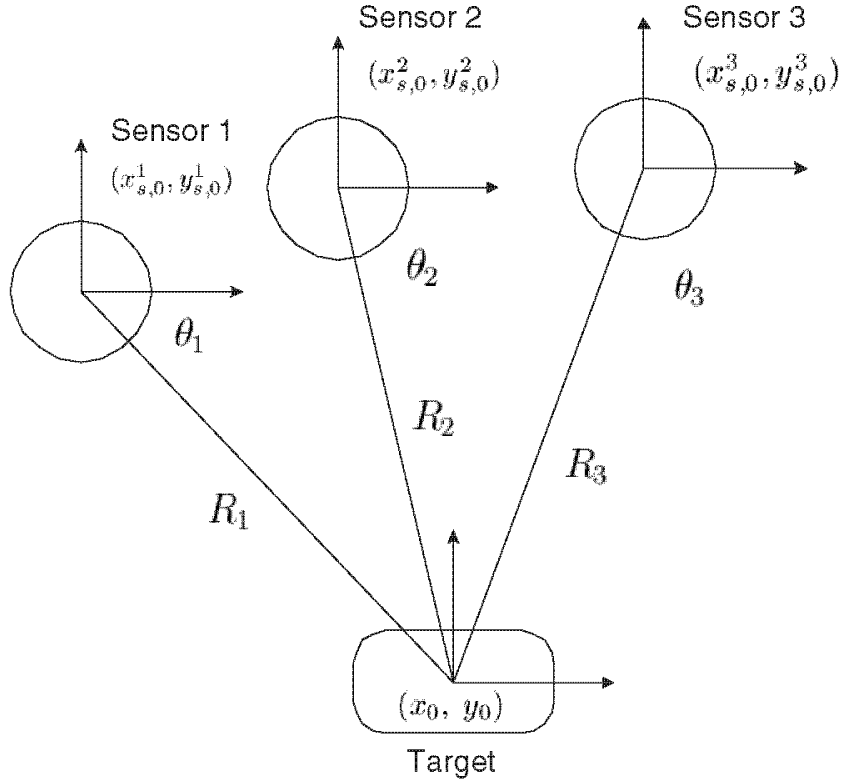

Fig. 1. Typical geometry for three Sensors tracking a target

discretize the filtering density $p\left(\mathbf{X}_{n} \mid \mathbf{Y}_{0: n}\right)$

$$
p\left(\mathbf{X}_{n} \mid \mathbf{Y}_{0: n}\right) \approx \sum_{i=1}^{N} w_{n}^{i} \delta\left(\mathbf{X}_{n}-\mathbf{X}_{n}^{i}\right)
$$

where $\delta$ denotes the dirac delta function. The particles are obtained in a recursive manner, at each time instance $n$ the particles of time instance $n-1$ are used in sampling from an importance function $\pi\left(\mathbf{X}_{n} \mid \mathbf{X}_{n}^{i}, \mathbf{Y}_{0: n}\right)$

$$
\mathbf{X}_{n}^{i} \sim \pi\left(\mathbf{X}_{n} \mid \mathbf{X}_{n}^{i}, Y_{0: n}\right) \text { for } 1 \leq i \leq N,
$$

the particle weighting coefficient $w_{n}^{i}$ is obtained by the calculation

$$
w_{n}^{i}=w_{n-1}^{i} \frac{p\left(Y_{n} \mid \mathbf{X}_{n}^{i}\right) p\left(\mathbf{X}_{n}^{i} \mid \mathbf{X}_{n-1}^{i}\right)}{\pi\left(\mathbf{X}_{n}^{i} \mid \mathbf{X}_{0: n}^{i}, Y_{0: n}\right)} .
$$

In order to initialize the particle filter, most work in the literature assumes a known initial state distribution $\mu\left(\mathbf{X}_{0}\right)$. Given this initial state distribution, the particle filter initializes its particles by sampling directly from this distribution and setting all weights to be equal to each other. This initialization procedure can be written,

$$
\begin{aligned}
\mathbf{X}_{0}^{i} & \sim \mu\left(\mathbf{X}_{0}\right) \\
w_{0}^{i} & =1 / N .
\end{aligned}
$$

Note that it is assumed that the initial distribution must be known exactly; in many applications this is not a valid assumption and the particle filter must be initialized from the first available measurement or other prior information. Good filter initialization allows the filter to lock into the target's track and quickly convergence to its steady state performance. 
In contrast, low quality initialization can degrade filter performance for the first time iterations. More importantly, incorrect filter initialization coupled with unavoidable minor modeling inaccuracies can cause filter divergence [1].

\section{NEW BEARING ONLY TRACKER INITIALIZATION}

The initialization scheme consists of several distinct steps. First, we process the $K$ available bearing measurements using a least squares strategy [5] that resolves the multiple bearing observations to obtain multiple position estimates. These estimates are in turn shifted to the target's coordinate system and used to estimate the statistics of the initial state distribution. The statistics estimation is performed straightforwardly by computing the mean and variance of the initial state distribution. Making the assumption that the initial state distribution is Gaussian, the mean and variance fully describe the initial state behavior. Thus, one obtains a full description of an approximate Gaussian initial state distribution.

Following [5], write the initial range and bearing of the target as $r_{i}$ and $\theta_{i}$ respectively, where the index $i$ denotes that these range and bearing are taken by the $i$-th sensor. The following trigonometric relations are true,

$$
\begin{aligned}
r_{i} \cos \theta_{i} & =x_{0}-x_{s, 0}^{i} \\
r_{i} \sin \theta_{i} & =y_{0}-y_{s, 0}^{i},
\end{aligned}
$$

for all sensors $1 \leq i \leq K$. By writing these relations for all sensors, one can eliminate the unknown initial coordinates $\left(x_{0}, y_{0}\right)$ and rewrite the trigonometric equations in matrix form,

$$
\mathbf{A}\left[r_{1} r_{2} \cdots r_{K}\right]^{T}=\mathbf{B}
$$

where

$$
\mathbf{A}=\left(\begin{array}{ccccc}
\cos \theta_{1} & -\cos \theta_{2} & 0 & \cdots & 0 \\
\sin \theta_{1} & -\sin \theta_{2} & 0 & \cdots & 0 \\
0 & \cos \theta_{2} & -\cos \theta_{3} & \cdots & 0 \\
0 & \sin \theta_{2} & -\sin \theta_{3} & \cdots & 0 \\
\vdots & \vdots & \vdots & & \vdots \\
0 & 0 & 0 & \cdots & -\cos \theta_{K} \\
0 & 0 & 0 & \cdots & -\sin \theta_{K}
\end{array}\right)
$$

and

$$
\mathbf{B}=\left(\begin{array}{c}
-x_{s, 0}^{1}+-x_{s, 0}^{2} \\
-y_{s, 0}^{1}+-y_{s, 0}^{2} \\
-x_{s, 0}^{2}+-x_{s, 0}^{3} \\
-y_{s, 0}^{2}+-y_{s, 0}^{3} \\
\vdots \\
-x_{s, 0}^{K-1}+-x_{s, 0}^{K} \\
-y_{s, 0}^{K-1}+-y_{s, 0}^{K}
\end{array}\right)
$$

This is an over-determined matrix equation since $A$ is not a square matrix, a single solution does not exist so one may opt for the standard least squares solution which minimizes the squared error. Let the symbol $\widehat{r}_{i}$ denote the range estimate at the coordinate system of the $i$-th sensor, then write the least squares solution as,

$$
\left[\widehat{r}_{1}, \widehat{r}_{2} \cdots \widehat{r}_{K}\right]^{T}=\left(\mathbf{A}^{T} \mathbf{A}\right)^{-1} \mathbf{A}^{T} \mathbf{B}
$$

Transfering these estimates to the target's coordinate system,

$$
\begin{array}{cl}
\widehat{\mathbf{X}}_{1} & =\left(\widehat{r}_{1} \cos \theta_{1}+x_{0, s}^{1}, \widehat{r}_{1} \sin \theta_{1}+y_{0, s}^{1}\right)^{T} \\
\widehat{\mathbf{X}}_{2} & =\left(\widehat{r}_{2} \cos \theta_{2}+x_{0, s}^{2}, \widehat{r}_{2} \sin \theta_{2}+y_{0, s}^{2}\right)^{T} \\
\vdots & \\
\widehat{\mathbf{X}}_{K} & =\left(\widehat{r}_{K} \cos \theta_{K}+x_{0, s}^{K}, \widehat{r}_{K} \sin \theta_{K}+y_{0, s}^{K}\right)^{T} .
\end{array}
$$

Therefore, there are $K$ estimates of the initial position of the initial target. Next, one can compute the sample mean and variance of these estimates as follows,

$$
\begin{aligned}
\mathbf{M}_{\mu} & =\frac{1}{K} \sum_{j=1}^{K} \widehat{\mathbf{X}}_{j} \\
\boldsymbol{\Sigma}_{\mu} & =\frac{1}{K-1} \sum_{j=1}^{K}\left(\widehat{\mathbf{X}}_{j}-\mathbf{M}_{\mu}\right)\left(\widehat{\mathbf{X}}_{j}-\mathbf{M}_{\mu}\right)^{T}
\end{aligned}
$$

Making the assumption that the initial state distribution $\mu\left(\mathbf{X}_{0}\right)$ is Gaussian, it is well known that the the sample mean (Eq. 11) and sample variance (Eq. 12) are maximum likelihood estimators of the mean and variance of the distribution $\mu\left(\mathbf{X}_{0}\right)$. Therefore, one can approximate the initial state distribution by writing $\mu\left(\mathbf{X}_{0}\right) \sim \mathcal{N}\left(\mathbf{M}_{\mu}, \mathbf{\Sigma}_{\mu}\right)$. Finally, an approximate form of the initial state distribution's density function is obtained. Although only two sensors are required for this procedure, it is clear the accuracy of the mean and variance estimations improve as more sensors are available since additional position estimates can be computed. Given the approximate initial state distribution, the particle filter is initialized by sampling from the distribution $\mu\left(\mathbf{X}_{0}\right)$,

$$
\begin{aligned}
\mathbf{x}_{0}^{i} & \sim \mathcal{N}\left(\mathbf{M}_{\mu}, \boldsymbol{\Sigma}_{\mu}\right) \\
w_{0}^{i} & =1 / N .
\end{aligned}
$$

Note that it is straightforward to incorporate the prior knowledge of the initial range used by the RPEKF initialization scheme into this particle filter initialization scheme. Denote the limits between which the initial range $r_{0}$ lies in as $r_{0} \in$ $\left(r_{0}^{\min }, r_{0}^{\max }\right)$ where $r_{0}^{\min }$ and $r_{0}^{\max }$ are the lower and upper limits respectively. Then one can modify the initial state distribution to use this information can using a truncated normal distribution. This distribution can be written,

$$
\mu\left(X_{0}\right)=\left\{\begin{array}{cl}
c \mathcal{N}\left(\mathbf{M}_{\mu}, \mathbf{\Sigma}_{\mu}\right) & \text { for } r_{0}^{\min } \leq \mu\left(X_{0}\right) \leq r_{0}^{\max } \\
0 & \text { otherwise }
\end{array}\right.
$$

where $c$ is an appropriate scalar value that turns $\mu\left(X_{0}\right)$ into a probability density. This truncated normal distribution can 
then be sampled from by the particle filter in a straightforward manner as in Equation (4). Also, if the prior information of the initial range is sufficiently accurate; one can modify the least squares procedure to a linearly constrained least squares procedure. In such a case, the constraint can be written

$$
r_{0}^{\min }[1,1 \cdots, 1] \leq\left[\widehat{r}_{1}, \widehat{r}_{2} \cdots \widehat{r}_{K}\right]^{T} \leq r_{0}^{\max }[1,1 \cdots, 1]
$$

in order to prevent any estimates from falling outside the allowed values for the initial range.

\section{SIMULATIONS}

We simulate a configuration of three stationary bearing only sensors. We compare the initial performance of the EKF initialized with the ground truth and a particle filter initialized using the new initialization scheme described above. The simulations are done by generating 50 Monte Carlo tracks with identical initial state and model parameters and performing the tracking on each one. The error metric used in these simulations is root mean square error (RMSE). The RMSE errors for each track are averaged to produce an overall initial performance graph. Figure 2 shows the RMSE performance

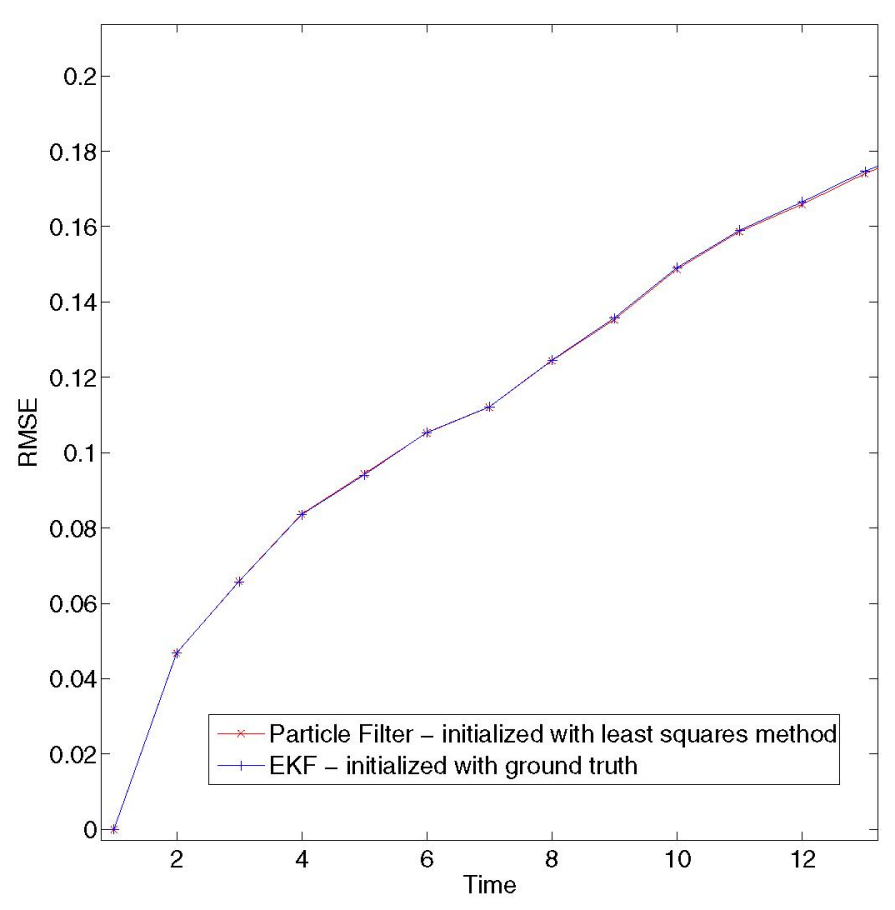

Fig. 2. Average performance of the EKF and Particle filter in Bearing Only Tracking.

of two trackers. The first tracker is a particle filter initialized using the new scheme developed above. This algorithm is compared against an extended Kalman filter which is initialized with the initial ground truth state. In other words, the EKF's initialization is optimal while the particle filter uses the non-perfect initialization. It is clear from Figure 2 that on average, the initialization of the particle filter produces the same initial performance as that of the EKF initialized with the true state. Therefore, we can conclude that on average, the initialization method suggested above performs as well as a tracker initialized with the true initial state of the target. This result shows very satisfactory performance by the initialization scheme that will prevent any tracker divergence due to initialization errors.

\section{CONCLUSION}

In this paper, a new initialization scheme is presented to initialize a particle filter tracker using multiple bearing observations. The tracking algorithm is a particle filter, this filter can approximate the true optimal filtering density for arbitrary nonlinear and non-Gaussian state space systems. The particle filter is initialized by the initial state distribution that is constructed using the available bearing observations. This state distribution is obtained by assuming it to be normal and calculating its mean and variance using multiple initial state estimates. The initial state estimates are calculated by a least squares procedure that resolves multiple bearings. Simulations indicate that on average, this initialization method is as good as initializing the filter with the true initial state. This good initial performance allows the particle filter to quickly stabilize and convergence to the track.

\section{REFERENCES}

[1] V.J. Aidala and S.E. Hammel, "Utilization of modified polar coordinates for bearing-only tracking", IEEE Trans. Automatic Control, vol. 28, no. 3, pp. 283-294, March 1983.

[2] N. Peach, "Bearings-only tracking using a set of range parameterised extended Kalman filters," Proc. Inst. Elect. Eng. Contr. Theory Appl., vol. 142, no. 1, Jan. 1995.

[3] A. Doucet, S.J. Godsill, and C. Andrieu, "On sequential Monte Carlo sampling methods for Bayesian filtering". Stat. \& Comp., vol. 10, no. 3, pp. 197-208, 2000.

[4] Y. Zhou, A Kalman Filter based Registration Approach for Multiple Asynchronous Sensors, Defense R\&D Canada - Ottawa, 2003.

[5] D. Koks, "Passive geolocation for multiple receivers with no initial state estimate", Electronic Warfare Division: Electronics and Surveillance Research Laboratory, DSTO. 